\title{
Complex Permeability After-Effect and Analysis of Power Losses in Ferromagnetic Co-Based Amorphous Alloy
}

\author{
J. KRAVČÁK \\ Department of Physics, FEI, Technical University of Košice \\ Park Komenského 2, 04200 Košice, Slovakia \\ R. VARGa AND P. VoJTANÍK \\ Institute of Physics, P.J. Šafárik University \\ Park Angelinum 9, 04154 Košice, Slovakia
}

\begin{abstract}
Complex permeability $\left(\mu=\mu_{1}-\mathrm{i} \mu_{2}\right)$ after-effect of near-zero magnetostrictive $\mathrm{CoFeCrSiB}$ amorphous alloy was investigated in magnetic field $H(t)=H_{0} \exp (\mathrm{i} 2 \pi f t)$ after demagnetization. For frequencies $f$ from $200 \mathrm{~Hz}$ to $2000 \mathrm{~Hz}$ and for the small amplitude $H_{0}<H_{\text {cr }}$ we observed practically constant both real $\mu_{1}$ and imaginary part $\mu_{2}$ of permeability. A measured small decrease in the real part $\mu_{1}$ and increase in the imaginary part $\mu_{2}$ with frequency $f$ were theoretically calculated for a quadratic form of a domain wall potential $E_{\mathrm{S}}(x)=\frac{1}{2} \alpha x^{2}$. The calculated loss factor $\tan \delta=\mu_{2} / \mu_{1}$, which is small at amplitude $H_{0}<H_{\mathrm{cr}}$, corresponds to power losses due to eddy currents induced around reversibly moving domain walls.
\end{abstract}

PACS numbers: 75.40.Gb, 75.60.Ch, 75.60.Lr

\section{Introduction}

After demagnetization procedure the positions of the domain walls (DWs) are determined by the static pinning potential $E_{0}(x)$ ( $x$ is a DW displacement). The magnetic after-effect (MAE) of permeability $\mu=\mu_{1}-\mathrm{i} \mu_{2}$ is related to the stabilization potential $E_{\mathrm{S}}(x, t)=E_{\mathrm{S}}(x) G(t)$, separated to $E_{\mathrm{S}}(x)=\frac{1}{2} \alpha x^{2}$, for small $x<d$ ( $d$ is a DW thickness) and to time relaxation function $G(t)$, when an external magnetic field $H(t)=H_{0} \exp (\mathrm{i} \omega t)$ with small amplitude $H_{0}<H_{\text {cr }}$ is applied. The MAE of the complex permeability $\mu$ can be expressed as a change of its reciprocal value (reluctivity) $r=1 / \mu=r_{1}+\mathrm{i}_{2}: \Delta r(t)=r\left(t_{2}\right)-r\left(t_{1}\right)$ at time interval from $t_{1}$ to $t_{2}$ after demagnetization [1]. 


\section{Theoretical model}

From the theoretical point of view it is a very interesting idea of Döring that the moving DW exhibits an inertia. The mass of the DW has its origin in the angular momenta of the spins forming the wall [2]. We consider the equation of motion for $180^{\circ} \mathrm{DW}$ :

$$
m \frac{\mathrm{d}^{2} x}{\mathrm{~d} t^{2}}+\beta \frac{\mathrm{d} x}{\mathrm{~d} t}+\alpha x=2 \mu_{0} M_{\mathrm{s}} H
$$

where $m$ is the mass of the DW per unit area, $\beta$ - the damping coefficient, $\alpha$ - the restoring coefficient. The term $2 \mu_{0} M_{\mathrm{s}} H$ on the right side of the equation represents the pressure acting on the $180^{\circ} \mathrm{DW} . H(t)=H_{0} \exp (\mathrm{i} \omega t)$ is applied with a small amplitude $H_{0}$ less than critical $H_{\mathrm{cr}}$, the solution of Eq. (1) can be found

$$
x(t)=\frac{2 \mu_{0} M_{\mathrm{s}} H_{0} / \alpha}{1-(m / \alpha) \omega^{2}+\mathrm{i}(\beta / \alpha) \omega} \exp (\mathrm{i} \omega t) .
$$

In the demagnetized state, the displacement of the $180^{\circ} \mathrm{DW} x$ is connected with the magnetic polarization: $J(t)=2 \mu_{0} M_{\mathrm{s}} x(t)$, and then the expression for complex permeability can be obtained

$$
\mu(\omega)=\mu_{1}-\mathrm{i} \mu_{2}=\frac{J(t)}{H(t)}=\frac{\mu_{\mathrm{s}} \exp (-\mathrm{i} \delta)}{\sqrt{\left[1-\left(\omega^{2} m / \alpha\right)\right]^{2}+(\omega \beta / \alpha)^{2}}}
$$

where $\mu_{\mathrm{s}}=4 \mu_{0}^{2} M_{\mathrm{s}}^{2} / \alpha$ states for static permeability. The loss factor is

$$
\tan \delta=\mu_{2} / \mu_{1}=\frac{\omega \beta / \alpha}{1-\omega^{2} m / \alpha} .
$$

\section{Experiment}

Near zero magnetostrictive amorphous alloy $\mathrm{Co}_{66.6} \mathrm{Fe}_{3.9} \mathrm{Cr}_{6.9} \mathrm{Si}_{7.6} \mathrm{~B}_{15}$ in as-cast state with the coercive field $H_{\mathrm{c}}=0.6 \mathrm{~A} \mathrm{~m}^{-1}$ was used in experiments. Ribbon-shaped sample $5 \mathrm{~mm}$ wide was used as a core of toroidal coil with the mean diameter of $22 \mathrm{~mm}$. The MAE was measured between $t_{1}=30 \mathrm{~s}$ and $t_{1}=300 \mathrm{~s}$ after demagnetization by an AC magnetic field decreasing in $5 \mathrm{~s}$ from maximum amplitude to zero [3].

\section{Results and discussion}

The dependences of real $\mu_{1}$ and imaginary $\mu_{2}$ parts of $\mathrm{Co}_{66.6} \mathrm{Fe}_{3.9} \mathrm{Cr}_{6.9} \mathrm{Si}_{7.6} \mathrm{~B}_{15}$ alloy on the amplitude of applied field $H$ exhibit perminvar behaviour (Fig. 1). For the amplitude $H_{0}<H_{\mathrm{cr}}=1.4 \mathrm{~A} \mathrm{~m}^{-1} \mathrm{DWs}$ remain localized. In case of periodic movement of DWs $x(t)=x_{0} \exp (\mathrm{i}(\omega t-\delta))$ forced by external driving field $H(t)=H_{0} \exp (\mathrm{i} \omega t)$, the $x_{0}$ is small, taking into account a ratio $H_{\mathrm{c}} / H_{\mathrm{cr}}=5$. We can find a small decrease in the real part $\mu_{1}$ with frequency $f=\omega / 2 \pi:\left[\mu_{1}(200 \mathrm{~Hz})-\mu_{1}(2000 \mathrm{~Hz})\right] / \mu_{1}(200 \mathrm{~Hz})=1.5 \%$ (Fig. 2a). In limit case $\omega \rightarrow 0, \mu_{1}$ goes to $\mu_{\mathrm{s}}=4 \mu_{0}^{2} M_{\mathrm{s}}^{2} / \alpha$. The imaginary part $\mu_{2}$ increases with the frequency $f$ (Fig. 2a). As it results from Eq. (3), $\mu_{2}$ is 

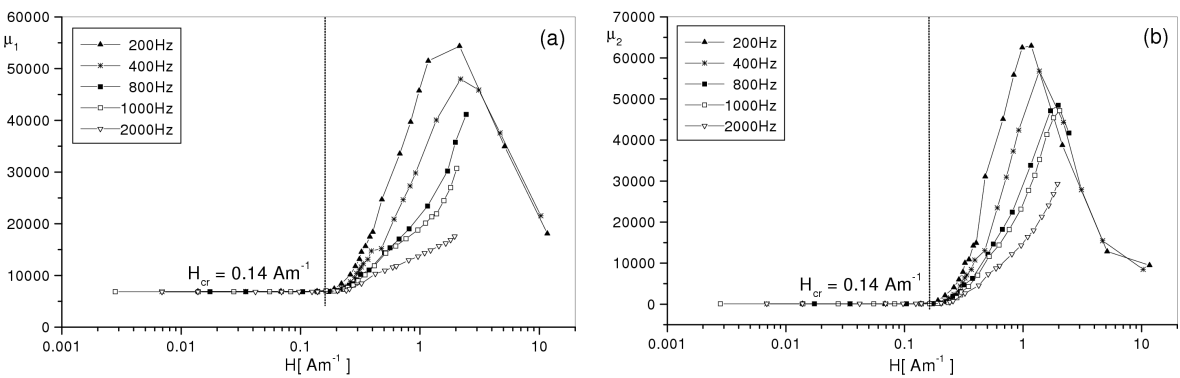

Fig. 1. (a) Dependence of the real part of permeability $\mu_{1}$ on amplitude of applied magnetic field. (b) Dependence of the imaginary part of permeability $\mu_{2}$ on amplitude of applied magnetic field. The frequencies of measurements and critical amplitude $H_{\mathrm{cr}}$ are displayed.
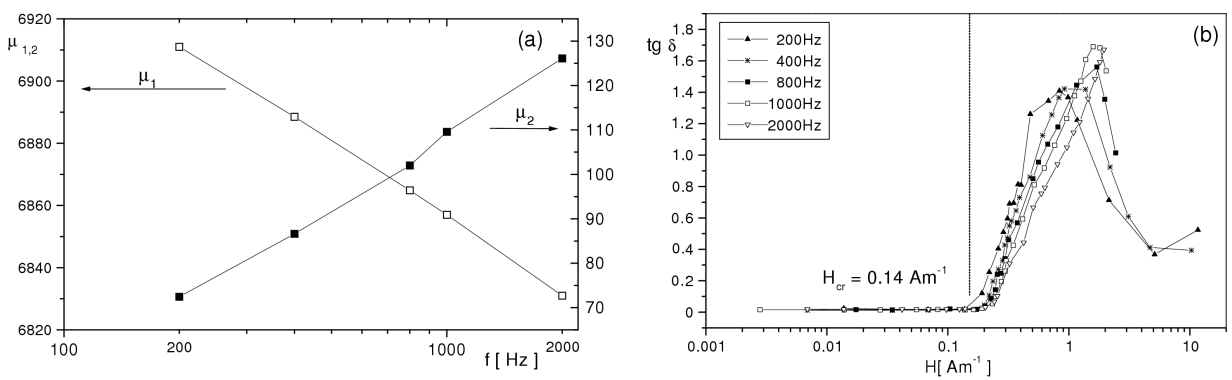

Fig. 2. (a) Real $\mu_{1}$ and imaginary $\mu_{2}$ part of permeability dependence on frequency of applied magnetic field, with amplitude less than critical $H_{\mathrm{cr}}$. (b) Dependence of the loss factor $\tan \delta=\mu_{2} / \mu_{1}$ on amplitude of applied magnetic field. The frequencies of measurements and critical amplitude $H_{\mathrm{cr}}$ are displayed.
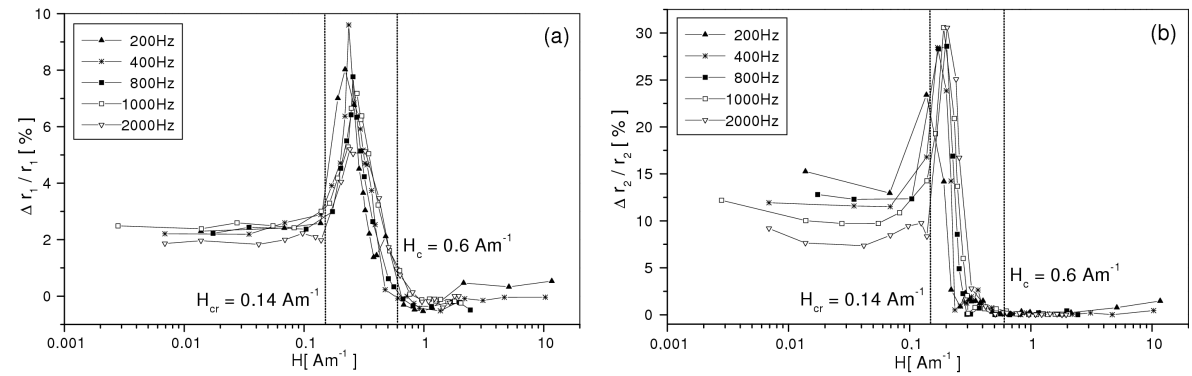

Fig. 3. Relative change of the real part $\Delta r_{1} / r_{1}$ (a) and imaginary part $\Delta r_{2} / r_{2}$ (b) of the reciprocal permeability (reluctivity) $r=1 / \mu$ on amplitude of magnetic field. The critical amplitude $H_{\mathrm{cr}}$ and coercivity $H_{\mathrm{c}}$ are displayed.

proportional to damping coefficient $\beta$ and goes to zero in limit case $\omega \rightarrow 0$. The calculated loss factor $\tan \delta=\mu_{2} / \mu_{1}$ (Eq. (4)), reflecting the power losses, increases with frequency similarly as $\mu_{2}$ (Fig. 2). In the range where $H_{\mathrm{cr}}<H_{0}$, 
we observe the rapid increase in permeability $\mu_{1}$ and $\mu_{2}$ (Fig. 1 ). The maxima of $\mu_{1}$ and $\mu_{2}$ are shifted towards higher field intensity and their values decrease with frequency. This indicates a substantial influence of frequency dependent eddy currents induced during the Barkhausen jumps. We register a huge increase in power losses connected with this irreversible DW movement (Fig. 2b). MAE of real part of reluctivity $\Delta r_{1} / r_{1}=2 \%$ in $H_{0}<H_{\text {cr }}$ (Fig. 3a). MAE of imaginary part of reluctivity $\Delta r_{2} / r_{2}$ decreases with frequency from $15 \%$ to $7 \%$ in $H_{0}<H_{\mathrm{cr}}$ (Fig. 3b). When amplitude $H_{0}$ exceeds critical $H_{\mathrm{cr}}$, we observe sharp peaks in MAE dependences of $\Delta r_{1} / r_{1}$ and $\Delta r_{2} / r_{2}$ and rapid decrease in both MAE dependences to zero in interval of amplitude $H_{\mathrm{cr}}<H_{0}<H_{\mathrm{c}}$ (Fig. 3 ).

\section{Conclusion}

There are two processes affecting the power losses in $H_{0}<H_{\mathrm{cr}}$ : (1) stabilization of the domain walls, (2) eddy currents induced during reversible periodic movement of domain walls. When amplitude $H_{0}$ exceeds critical $H_{\mathrm{cr}}$, the destabilization of domain walls occurs starting with irreversible Barkhausen jumps followed by maximum of MAE.

\section{Acknowledgments}

This work was partly supported by scientific grant VEGA No. 1/3035/06.

\section{References}

[1] H. Kronmüller, Philos. Mag. B 48, 127 (1983).

[2] S. Chikazumi, Physics of Magnetism, Wiley, New York 1964.

[3] P. Vojtaník, J. Kravčák, R. Varga, J. Magn. Magn. Mater. 157/158, 175 (1996). 